\title{
The problematic connection between gamma-ray bursts and ultra-high energy cosmic rays
}

\section{Damien Bégué*}

Max-Planck Institute for Extraterrestrial Physics, Garching, Germany

E-mail: dbegue ampe.mpg. de

\section{Filip Samuelsson}

KTH Royal Institute of Technology, Stockholm, Sweden E-mail: filipsamekth. se

\section{Asaf Pe'er}

Bar-Ilan University, Ramat Gan, Israel E-mail: asaf.peer@biu.ac.il

\section{Felix Ryde}

KTH Royal Institute of Technology, Stockholm, Sweden E-mail: fryde@kth. se

\begin{abstract}
The acceleration site for UHECR is still an open question despite extended research and GRBs are considered one of the most promising source candidates. Under the likely assumption that electrons are also accelerated at the UHECR acceleration site, synchrotron emission from these co-accelerated electrons is inevitable. We characterize this synchrotron emission and compare it to observed GRB spectra and find that for standard parameters, the synchrotron flux from these electrons would be much too luminous. This result challenges both high- and low-luminosity GRBs as accelerators of UHECR. A detailed discussion on GRB 060218 as UHECR source is also presented.
\end{abstract}

High Energy Phenomena in Relativistic Outflows VII - HEPRO VII

9-12 July 2019

Facultat de Física, Universitat de Barcelona, Spain

\footnotetext{
* Speaker.
} 


\section{Introduction}

The production site of ultra-high energy cosmic rays (UHECR), with energy $E>10^{18.5} \mathrm{eV}$ is still uncertain and highly debated. Gamma-ray bursts have been considered a promising candidate for the production of such particles [1]. Indeed, when considering the cooling efficiency of ions and the total power output, it is found that GRBs could explain the ultra-high energy cosmic rays up to the highest observed energies of a few $10^{20} \mathrm{eV}$, see e.g. [2, 3]. Here, we discuss constraints that can be put on the maximum cosmic ray energy by using the spectral properties of the prompt phase under the guise of non-thermal synchrotron radiation models and photospheric emission models. These results alongside with more details, specifically on the influence of the parameters (not discussed in this proceeding), can be found in $[4,5]$.

Gamma-ray bursts are cosmological cataclysmic events of extreme luminosity associated to the death of massive star or the merger of two compact objects (neutron stars and black-hole), for a review see e.g. [6,7]. Observationally, their emission is composed of two episodes: the prompt phase and the afterglow. The latter is due to the interaction of an ultra-relativistic ejecta with the surrounding medium, while the origin of the former is still highly uncertain. Main contenders are photospheric emission produced when a relativistic jet becomes transparent [8], non-thermal synchrotron radiation from electrons accelerated in shocks or magnetic reconnection, see e.g. [9, $10,11]$.

Observationally, the prompt phase is characterised by a high gamma-ray flux in the few of tens $\mathrm{keV}$ to few $\mathrm{MeV}$ energy band. Spectral analysis indicate that the origin of the emission is either photospheric emission $[12,13]$ or marginally fast cooling synchrotron radiation with $\gamma_{m} \sim \gamma_{c}{ }^{1}$ $[14,15]$. We consider both models in turn and set constrains for both of them. This proceeding is organised as follow. First, we review the main cooling processes of ions, then we consider the implication for non-thermal models and then for thermal models. Finally, we specifically discuss GRB 060218.

\section{Cooling and acceleration rates}

The cooling of ions is mainly due to adiabatic expansion, synchrotron cooling and photohadronic interactions. The latter is computed assuming the characteristics of an average GRB, i.e. with luminosity $L=10^{52} \mathrm{erg} . \mathrm{s}^{-1}$ and peak energy $\varepsilon=300 \mathrm{keV}$. In any case, since the photohadronic process is relevant only small radii where the photon and proton densities are large, the exact values of $L$ and $\varepsilon$ do not influence the final results. Other cooling mechanisms such as BetheHeitler are not discussed. If such processes would be relevant, it would make our results harsher. The relevant expressions for the cooling rate and discussion on the assumption can be found in [4]. We note that heavy elements are photo-dissociated in GRB jets because of the intense radiation field [16]. However, iron could in principle subsist in low-luminosity GRB jets. We discuss the acceleration of iron specifically for GRB 060218 in the last section.

\footnotetext{
${ }^{1}$ Here $\gamma_{c}$ is the electron cooling Lorentz factor while $\gamma_{m}$ is the injection Lorentz factor.
} 

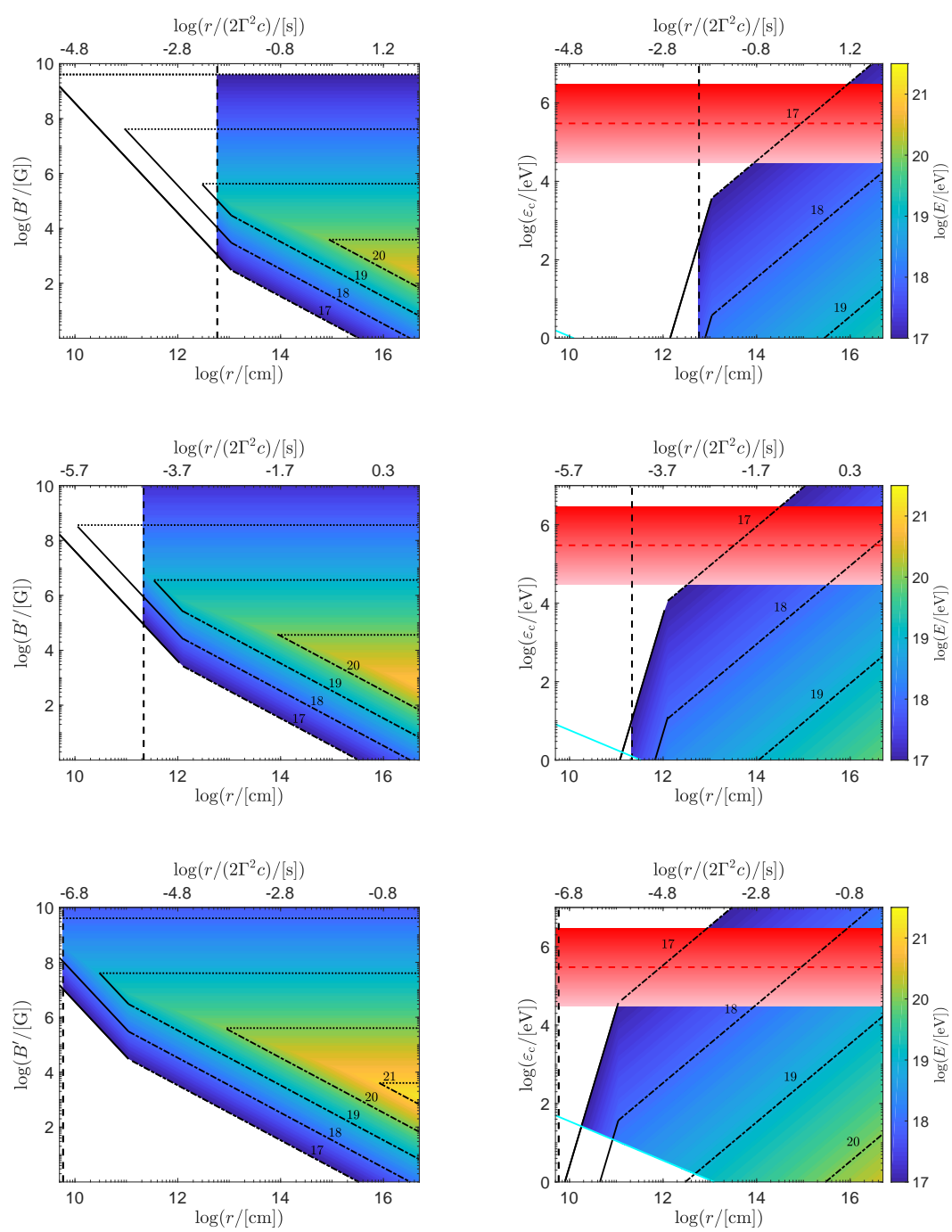

Figure 1: Left: Maximum UHECR energy for protons as a function of comoving magnetic field and radius for Lorentz factor 100 (top), 300 (middle) and 1000 (bottom). Right: Maximum UHECR energy as a function of observed synchrotron cooling frequency for the corresponding Lorentz factor. The vertical dashed line corresponds to the photospheric radius, below which particles cannot be accelerated. The red band corresponds to the range of observed synchrotron cooling frequency (between $\sim 30 \mathrm{keV}$ to $\sim 3 \mathrm{MeV}$ ) and the red dashed line is the average peak energy at $300 \mathrm{keV}$. From [4].

The acceleration timescale considered is that for diffusive shock acceleration for a strong shock given by

$$
t_{\mathrm{acc}}^{\prime}=\frac{E}{\eta c Z_{i} e B^{\prime} \Gamma}
$$

where primed quantities are expressed in the comoving frame, $E$ is the observed UHECR energy, $Z_{i}$ its charge, $B$ is the magnetic field strength, $e$ and $c$ are the elementary charge and the speed of light and $\Gamma$ is the bulk Lorentz factor. In addition, $\eta \sim 0.1$ is a normalization constant which scales the acceleration efficiency. It cannot be larger than the unity, else requiring the Larmor radius to 
be smaller than the system size becomes a harsher constrain than requiring the dynamical time to be smaller than $t_{\mathrm{acc}}^{\prime}$. From the cooling rates, the dynamical time and the acceleration rate, one can obtain the maximum observed particle energy as a function of comoving magnetic field and radius. We show the parameter space on the left side of Figure 1 for protons and for different Lorentz factors. We can see that there is a large parameter space which allows for particles to be accelerated to energy as large as few $10^{20} \mathrm{eV}$, thus satisfying the maximum observed cosmic ray energy.

\section{Results}

\subsection{Synchrotron models}

We now assume that the prompt emission is due to synchrotron radiation. As pointed out in [14], such emission models for GRBs must be characterised by marginally fast cooling with $\gamma_{c} \sim \gamma_{m}$. It is clear that the acceleration of high energy particles to $10^{20} \mathrm{eV}$ requires a large magnetic field. That also means that electrons, which are lighter than protons, can cool very efficiently. Calculating the cooling Lorentz factor of electrons, we find that acceleration of UHECR requires $\gamma_{c} \ll \gamma_{m}$, which translates in frequency to $v_{c} \ll v_{m}$ ( $v_{c}$ and $v_{m}$ are the cooling and injection frequency). We show in the right of Figure 1 the observed cooling frequency as a function of radius and corresponding maximum UHECR. The red band corresponds to the observed $v_{c}$ in the guise of synchrotron models. It is clear that the observational constraint $v_{c} \sim v_{m}$ reduces the maximum observed energy of UHECRs to be smaller than few $10^{18} \mathrm{eV}$. This result holds when $\eta$ is increased to its maximum value 1 .

\subsection{Photospheric models}

In the context of photospheric emission models, we constrain all dissipation scenario and associated non-thermal emission taking place above the photosphere. For this, we need to make several assumptions. First, all the $\mathrm{MeV}$ radiation comes from the photosphere. This sets a flux limit on the synchrotron radiation from co-accelerated electrons. Second, we consider observational limits on the optical flux from GRBs. We take $F_{\text {opt }}=10 \mathrm{mJy}$ and we consider an average redshift to be $z=1$. Third, we need to consider the fraction of energy used to accelerate electron $\varepsilon_{e} \sim 0.1$ and the fraction of electrons which are accelerated $\xi_{a} \sim 1$. We then consider all emission regimes, taking only the least constraining of them in terms of maximum UHECR energy. The constraints are set by the fluxe constraints in the $\mathrm{MeV}$ and optical band. The results are displayed in Figure 2. One can see that the maximum UHECR energy that can be reached is only few $10^{19} \mathrm{eV}$. A complete discussion on the influence of the different parameters can be found in [4].

\section{GRB 060218 as a low-luminosity GRB}

We now repeat this study for the specific GRB 060218. This GRB is a member of the hypothetical class of low-luminosity GRBs, defined by the small luminosity of their prompt phase $L<10^{48} \mathrm{erg} . \mathrm{s}^{-1}$. It is also believed that the Lorentz factor of their outflow is much smaller than that of classical long GRBs with typical expected values around $\Gamma \sim 10$. We present our results for $\Gamma=3$ and $\Gamma=10$. 

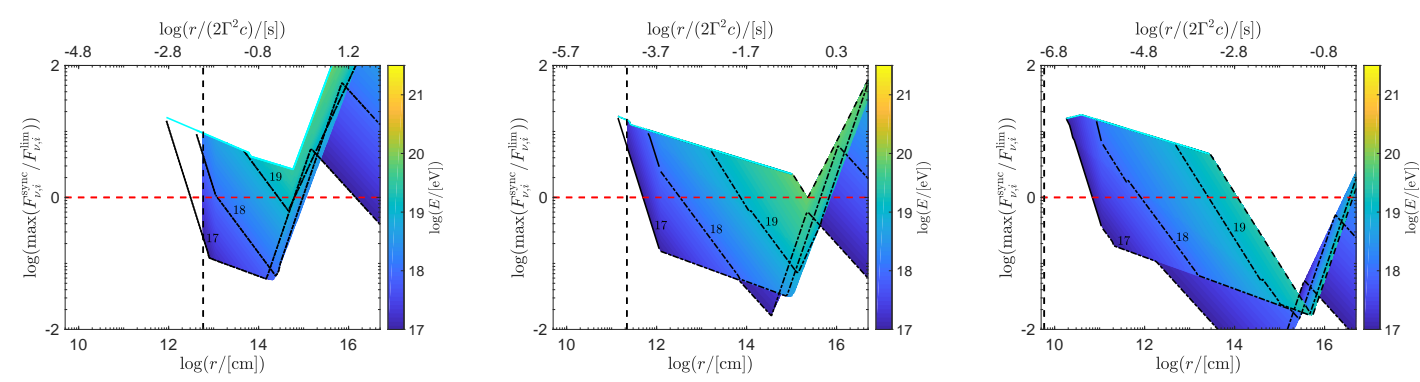

Figure 2: Limits on the maximum energy of UHECR for different Lorentz factor: 100 (left), 300 (middle) and 1000 (right). The parameter space above the red line would over-shine the limits either in the optical band or in the $\mathrm{MeV}$ band. The blue line corresponds to the limit of non-relativistic $\gamma_{c}$. From [4].

It is also widely believed that iron can survive in the outflow of low-luminosity because of the low luminosity of these events ${ }^{2}$. In what follow, we will constrain iron only, since if acceleration of iron is possible, so is the acceleration of lighter ions. In addition, GRB 060218 is often considered as a canonical GRB which can accelerate UHECR [17, 19]. For the specific purpose of this analysis, it is important to note that there are optical observations during the prompt phase of GRB 060218. These optical observations appear to allow for right constraints on the maximum energy of UHECR.

The top of Figure 3 shows the maximum iron energy that can be achieved for the fiducial parameters $\varepsilon_{e}=0.1, \xi_{a}=1$ and $\eta=1$ (corresponding to the highest possible acceleration rate). We see that there is no possibility for iron to be accelerated above $10^{19} \mathrm{eV}$. Yet, because the Lorentz factor of the outflow in low-luminosity GRB is small, the uncertainty on the parameters $\xi_{a}$ and $\varepsilon_{e}$ is large, and they can substantially vary. Numerical simulations of non-relativistic shocks indicates that $\varepsilon_{e}$ and $\xi_{a}$ can be as low as few $10^{-4}$ and $10^{-3}$. On the bottom panel of Figure 3, we show the most optimistic UHECR energy as a function of $\xi_{a}$ for $\varepsilon_{e}=5 \times 10^{-4}$. It is clear from the figures that 060218 cannot accelerate iron above few $10^{19} \mathrm{eV}$.

\section{Conclusion}

By considering the spectral properties of the prompt phase of GRBs, we constrained the maximum energy of UHECR that can be obtained in GRB prompt phase. We find that for both nonthermal synchrotron models and photospheric emission models, GRBs fall short to accelerate particles to energy larger than a few $10^{19} \mathrm{eV}$. We then considered the case of hypothetical low-luminosity GRBs and specifically of GRB 060218 and came to the conclusion that optical observations during its prompt phase set tight limits on the maximum energy achievable by UHECR iron. In the most optimistic case, it is difficult to obtain iron above few $10^{19} \mathrm{eV}$. We thus conclude that the prompt phase of GRBs under the guise of both synchrotron and photospheric models, is unlikely to dominate the UHECR observed up to energies of few $10^{20} \mathrm{eV}$. A more comprehensive presenta-

\footnotetext{
${ }^{2}$ However, we note the recent studies [18, 19] in which it was shown that even for low-luminosity GRBs, a photonuclear cascade can develop in the context of the internal shock model of GRBs, thus reducing the iron fraction in their outflow. This result is not incompatible with our study, since we constrain the maximum energy of the accelerated iron and not their fraction.
} 

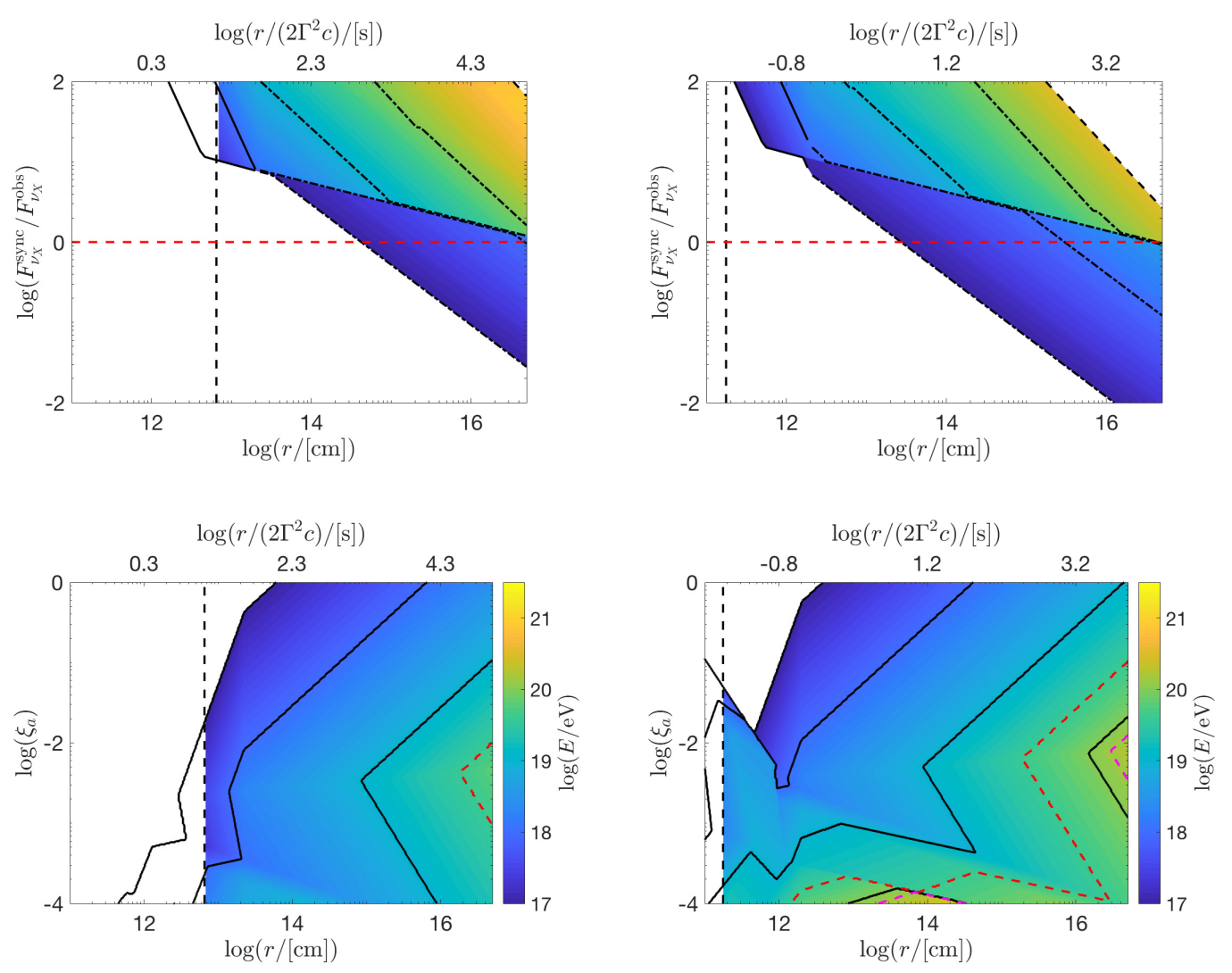

Figure 3: Left: $\Gamma=3$. Right $\Gamma=10$. Top: Same as Figure 2 but for iron. Bottom: highest possible energy that an iron can reach when changing $\xi_{a}$ for $\varepsilon_{e}=5 \times 10^{-4}$ and $\eta=1$. The thick black lines label each power of 10 of the observed particles energy. The red dashed line and magenta dashed line correspond to the maximum energy sufficient to fit the UHECR spectrum as found by [20] and [21]. From [5].

tion of these results together with a detailed discussion on the influence of the parameters and the limitations of the considered models can be found in [4] and [5].

\section{References}

[1] Waxman, E. 1995, Physics Review Letters, 75, 386

[2] Bustamante, M., Baerwald, P., \& Winter, W. 2013, ArXiv e-prints, arXiv:1306.2755

[3] Globus, N., Allard, D., Mochkovitch, R., \& Parizot, E. 2015, Mon. Not. R. Astron. Soc., 451, 751

[4] Samuelsson, F., Bégué, D., Ryde, F., \& Pe'er, A. 2019, arXiv e-prints, arXiv:1810.06579

[5] Samuelsson, F., Bégué, D., Ryde, F., \& Pe’er, A. 2020, in preparation.

[6] Mészáros, P. 2006, Reports on Progress in Physics, 69, 2259

[7] Pe'er, A. 2015, Advances in Astronomy, 2015, 907321

[8] Paczynski, B. 1986, Astrophys. J., 308, L43

[9] Rees, M. J., \& Meszaros, P. 1994, Astrophys. J., 430, L93 
[10] Daigne, F., \& Mochkovitch, R. 1998, Mon. Not. R. Astron. Soc., 296, 275

[11] Zhang, B., \& Yan, H. 2011, Astrophys. J., 726, 90

[12] Ahlgren, B., Larsson, J., Nymark, T., Ryde, F., \& Pe'er, A. 2015, Mon. Not. R. Astron. Soc., 454, L31

[13] Ryde, F., Lundman, C., \& Acuner, Z. 2017, Mon. Not. R. Astron. Soc., 472, 1897

[14] Burgess, J. M., Bégué, D., Bacelj, A., et al. 2018, arXiv e-prints, arXiv:1810.06965

[15] Oganesyan, G., Nava, L., Ghirlanda, G., Melandri, A., \& Celotti, A. 2019, Astron. Astrophys., 628, A59

[16] Horiuchi, S., Murase, K., Ioka, K., \& Mészáros, P. 2012, Astrophys. J., 753, 69

[17] Zhang, B. T. and Murase, K. and Kimura, S. S. and Horiuchi, S. and Mészáros, P. 2017, arXiv e-prints, arXiv:1712.09984

[18] Biehl, D., Boncioli, D., Fedynitch, A., \& Winter, W. 2018, Astron. Astrophys., 611, A101

[19] Boncioli, D., Biehl, D., \& Winter, W. 2018, Astrophys. J., 872, 110

[20] Heinze, Jonas and Fedynitch, Anatoli and Boncioli, Denise \& Winter, Walter, 2019, Astrophys. J., 873,88

[21] Aab, A., Abreu, P., Aglietta, M., Samarai, I. Al et al., 2017, JCAP, 4038 